\title{
Detection and Characterization of Carboxylic Acid Amide-Resistant Plasmopara viticola in China Using a TaqMan-MGB Real-Time PCR
}

\author{
Xiaoqing Huang, ${ }^{1}$ Xina Wang, ${ }^{1}$ Fanfang Kong, ${ }^{1}$ Theo van der Lee, ${ }^{2}$ Zhongyue Wang, ${ }^{1, \dagger}$ and Hao Zhang ${ }^{1, \dagger}$ \\ ${ }^{1}$ State Key Laboratory for Biology of Plant Diseases and Insect Pests, Institute of Plant Protection, Chinese Academy of \\ Agriculture Sciences, Beijing, China \\ ${ }^{2}$ Wageningen Plant Research, P. O. Box 16, 6700 AA Wageningen, The Netherlands
}

\begin{abstract}
Grape production is increasing globally and so are problems with downy mildew, one of the main constraints in grape production. Downy mildew on grape is caused by Plasmopara viticola, an obligate biotrophic pathogen belonging to the oomycetes. Control of the disease is usually performed by fungicide applications, of which carboxylic acid amide (CAA) fungicides represent one of the most widely used groups of fungicides. Our previous research showed that the extensive application of CAA fungicides can result in fungicide resistance and in China, CAAresistant isolates of $P$. viticola were collected from the field in 2014. To monitor the distribution and spread of CAA fungicide resistance, we developed a TaqMan-minor groove binder (MGB) real-time PCRbased method designed on a functional mutation in the PvCesA 3 gene

survey comprising 2,227 single-sporangiophore isolates from eight major grapevine regions in China. We demonstrate that CAA fungicide resistance in $P$. viticola is widespread in China. On average, $53.3 \%$ of the isolates were found to be resistant, but marked differences were found between locations with percentages of resistant isolates varying from 0.3 to $96.6 \%$. Furthermore, the frequency of CAA-resistant isolates was found to be significantly correlated with the exposure to CAA fungicides $(P<$ $0.05)$. We further discussed the possibilities to apply the TaqMan-MGB real-time PCR assay to assess the frequency of fungicide-resistant $P$. viticola isolates in each region or vineyard, which would facilitate the correct choice of fungicide for grape downy mildew and resistance management strategies.
\end{abstract} that allows efficient identification of CAA fungicide resistant and sensitive genotypes. The assay was validated on 50 isolates using Sanger sequencing and fungicide bioassays and exploited in a comprehensive
Keywords: Plasmopara viticola, TaqMan-MGB real-time PCR, carboxylic acid amide fungicides, resistance
Grape (Vitis vinifera L.) is one of the earliest domesticated and major economic fruit crops in the world (Myles et al. 2011). Since 2010, China is the largest grape producer in the world, and the total area of grape cultivation increased to 870,000 ha in 2017 , comprising table, wine, and dried grape (Tian et al. 2019). Table grape including V. vinifera, $V$. labrusca, and interspecific hybrids is most popular, covering $83 \%$ of the grape acreage (OIV 2018; Tian et al. 2019), followed by wine grape $(11 \%)$. Most wine grape varieties are $V$. vinifera, as well as some Chinese wild species, such as $V$. davidii and $V$. amurensis. One of the main constraints in grape production is downy mildew. This devastating disease is caused by Plasmopara viticola and has been implicated in major economic losses on a global scale (Emmett et al. 1992). In China, yield losses caused by grape downy mildew were reported ranging from 20 to $80 \%$ (Hu et al. 2013).

$P$. viticola is an obligate biotrophic pathogen that belongs to the oomycetes. Although they share a range of morphological features with fungi, oomycetes possess various distinct characteristics including a diploid vegetative mycelial stage and cellulose as a major

${ }^{\dagger}$ Corresponding authors: Z. Y. Wang, wangzhy0301@ sina.com; and H. Zhang, zhanghao@caas.cn

\section{Q. Huang and X. N. Wang contributed equally to this work.}

Funding: This work was supported by the National Key Research and Development Program of China (2018YFD0201309), the China Agricultural Research System (CARS-29), and Central Public-interest Scientific Institution Basal Research Fund (S2019XM04).

*The $\boldsymbol{e}$-Xtra logo stands for "electronic extra" and indicates that one supplementary table and one supplementary figure are published online.

The author(s) declare no conflict of interest.

Accepted for publication 31 March 2020.

(C) 2020 The American Phytopathological Society component of their cell wall. Downy mildew was first reported in China in 1899 and can be observed in almost all Chinese grape vineyards (reviewed by Yin et al. 2014). Although some American and Chinese wild species show different levels of resistance, all major commercial $V$. vinifera cultivars are highly susceptible to $P$. viticola (Yu et al. 2012) and chemical control is currently the most effective measure used to protect grapes from downy mildew disease (Chen et al. 2007; Waard et al. 1993).

Carboxylic acid amide (CAA) fungicides (FRAC code no. 40) are widely used to control the pathogens in the families Peronosporaceae (e.g., P. viticola and Bremia lactucae) and Pythiaceae (e.g., Phytophthora spp., but not Pythium spp.) (Gisi and Sierotzki 2008; Gisi et al. 2007a). CAA fungicides comprise a series of popular fungicides, such as dimethomorph, mandipropamid, pyrimorph, iprovalicarb, benthiavalicarb, valifenalate, and flumorph, all targeting the cellulose synthase 3 (CesA3) enzyme (Blum et al. 2010a). Resistance in $P$. viticola field populations to CAAs has been reported in Europe for almost 10 years, with cross-resistance shown between representatives of this class of fungicides (FRAC 2017; Gisi et al. 2007b). The implementation of effective management strategies impeding the evolution of resistance requires an accurate understanding of the emergence and development of resistance in pathogen populations (REX Consortium 2013; Schnabel et al. 2012). In China, dimethomorph was widely used to control $P$. viticola in the last 20 years, and CAA-resistant $P$. viticola isolates were first collected in 2014 (Zhang et al. 2017). However, the information on frequency of resistant isolates in China is still largely unknown.

As $P$. viticola is an obligate biotrophic pathogen, screening for fungicide-resistant isolates is traditionally performed using a sensitivity assay on grape leaf disks (Wong and Wilcox 2000). However, this method is laborious and time consuming. In 2010, Blum et al. first described the molecular mechanism underlying resistance to a CAA fungicide. Resistance to CAA is conferred by recessive mutations of the nuclear gene encoding cellulose synthase $3(P v C e s A 3)$. If an SNP leading to a substitution of a glycine (GGC) to a serine (AGC) residue at position 1105 (G1105S) in the deduced amino acid sequence is present in both alleles, isolates are resistant to CAA 
(Blum et al. 2010b). A second mutation at the same codon in $P v C e s A 3$ gene, G1105V, was also found associated with CAA resistance (Sierotzki et al. 2011), but it is detected at a low frequency and at a small number of sites (reviewed by Zhang et al. 2017). Based on the mutation G1105S of the PvCesA3 gene, several molecular methods have been developed for the detection of resistant isolates (Aoki et al. 2011, 2013; Zhang et al. 2017).

In our previous research, a tetra-primer amplification refractory mutation system (tetra-primer ARMS PCR) was established (Zhang et al. 2017). A set of four primers was designed to distinguish the different allelic variants (the homozygous and heterozygous alleles) in a single assay, which was demonstrated to be more rapid, accurate, and practical to detect $P$. viticola resistant to CAA fungicides compared with the traditional bioassays and some other molecular methods, such as PCR-RFLP (Zhang et al. 2017). Nevertheless, two-pair primers can affect the amplification specificity (Zhang et al., unpublished data)—such difference in primer melting temperature (Tm) was previously reported (Hamajima et al. 2002). A new strategy for diagnostics that is more practical for high throughput screening is therefore required. A TaqMan minor groove binder (MGB) probe real-time PCR assay was selected to enhance the specificity and accuracy of detection.

Application of a TaqMan-MGB probe was first reported in 2000 (Kutyavin et al. 2000). The MGB stabilizes A/T rich duplexes and allows use of shorter probes with higher melting temperature compared with ordinary DNA probes, hence increasing the specificity of an MGB probe and allowing discrimination of single-base mismatches (Ma et al. 2013; Van Hoeyveld et al. 2004). Furthermore, different TaqMan-MGB probes can be labeled with different fluorescence reporter dyes, which allows detection of multiple allelic variants in a single assay (Fan et al. 2014). In order to efficiently and simultaneously detect the two alleles of diploid organisms such as $P$. viticola, real-time PCR based on TaqMan-MGB probes is a particularly appropriate method.

In this study, a TaqMan-MGB real-time PCR based method was developed to detect the CAA fungicide resistant allele of $P$. viticola, based on a previously reported functional point mutation at codon
1105 of the $P v C e s A 3$ gene. Using this new method, a comprehensive survey of 2,227 isolates from eight grape-growing regions in China was performed to detect the frequency of resistance genotype to CAA fungicides. Its application to improve the integrated pest management strategies against $P$. viticola in grapevine fields in China is also discussed.

\section{Materials and Methods}

Isolates. The dimethomorph resistant isolate YQ1 and the sensitive isolate LF1, previously characterized by Zhang et al. (2017) using the leaf disc bioassay and Sanger sequencing, were used to test the specificities of the primers and probes of the newly developed TaqMan-MGB based assay. During the growing season in June to August of 2016, at the veraison and fruit maturation stage, $P$. viticola infected leaves were collected at random in each vineyard from plants along two crossing diagonal transects, and a total of 3,026 samples were obtained in eight major grape-growing regions, namely Beizhen, Qingxu, Wei, Binchuan, Penglai, Gongan, Huaihua, and Yongren, in China (Fig. 1). Singlesporangiophore isolations were conducted according to the method described by Zhang et al. (2017). Single-sporangiophore isolates were propagated on the sterilized leaf discs of $V$. vinifera 'Lizamate' on a water agar $(0.8 \%)$ medium in a Petri dish and incubated at $21^{\circ} \mathrm{C}$ with $16 \mathrm{~h}$ day and $8 \mathrm{~h}$ night at $100 \%$ humidity to ensure leaf wetness. After 7 days, leaf discs with sporangia and sporangiophores were immediately frozen in liquid nitrogen and stored at $-80^{\circ} \mathrm{C}$ for CAA resistance testing. A total of 2,277 single-sporangiophore strains were successfully isolated (Table 1). Fifty $P$. viticola isolates from Wei County were selected to validate the method (Table 2).

DNA extraction. DNA of the reference strains (YQ1 and LF1) was extracted using the SP Fungal DNA isolation kit (Omega, Norcross, GA) according to the manufacturer's protocol. DNA extraction of leaves with sporangia and sporangiophores of 2,227 strains was performed using the method described by Xin et al. (2003). DNA concentrations were determined by NanoVue Plus (GE Healthcare Life Sciences, Pittsburgh, PA).

TaqMan-MGB real-time PCR based method for detection of dimethomorph resistance. Design of TaqMan probes and primers.

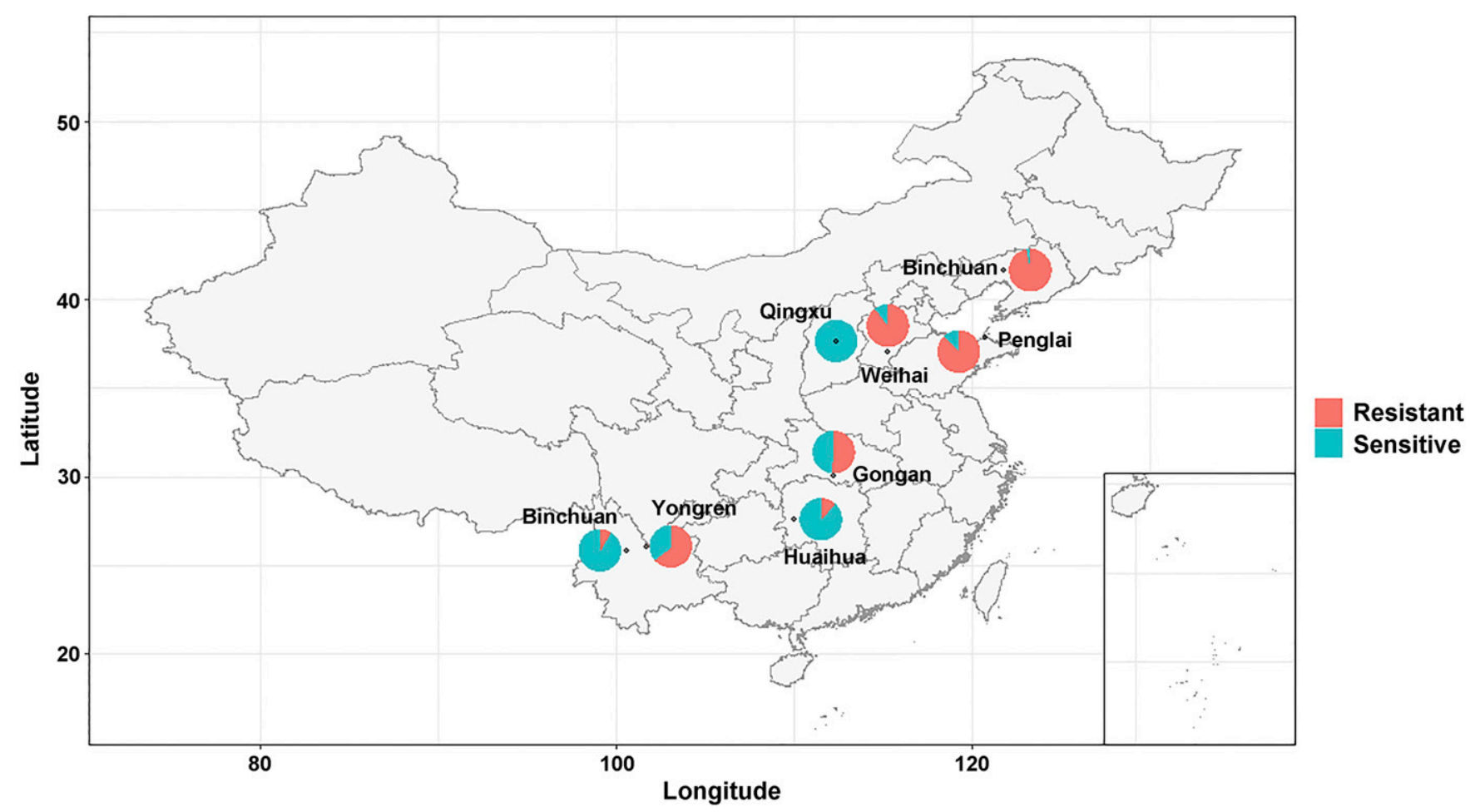

Fig. 1. The carboxylic acid amide (CAA) resistance and sensitive genotype frequency of $P$. viticola in the eight grape growing areas of China. The orange and green piece represents the percentage of CAA fungicide resistant and sensitive genotype strains, respectively, which were detected by the newly developed TaqMan-minor groove binder (MGB) real-time PCR based method. The black dot represents the sampling site. The map was generated in R (version 3.6.1). 
The $\mathrm{G}$ to A mutation at codon 1105 of the $P v C e s A 3$ gene causing a substitution of serine to glycine in the protein confers CAA resistance in P. viticola isolates (Blum et al. 2010b). Based on the alignment of PvCesA3 gene sequences (GQ258975.1, KU860541.1, KU860539.1, KU860536.1, KU860542.1, KU860540.1, KU860535.1, and KU860534.1), a pair of specific primers (Cesa3F/Cesa3R) was developed to amplify a region of $P v C e s A 3$ gene including codon 1105 using the Primer 5.0 software (PREMIER Biosoft, PaloAlto, CA). Two specific TaqMan probes (Cesa3Pr/Cesa3pS) targeting the $\mathrm{G}$ and A alleles, respectively, were designed by Primer Express 3.0 software (Applied Biosystems, Foster City, CA). Figure 2 shows the sequence targeted by the specific TaqMan MGB probes and primers. Information on the primers and probes used in this study is given in Table 3. The specific MGB TaqMan probes for the resistant (Cesa3pR) and the sensitive allele (Cesa3pS) were labeled with FAM (carboxyfluorescein) and VIC (Applied Biosystems, Carlsbad, CA) reporter dyes, respectively, at the $5^{\prime}$ end, and a nonfluorescent quencher (NFQ) with MGB ligands was used as the quencher at the $3^{\prime}$ end. The primers and probes were synthesized by Sangon Biotech (Shanghai, China) and Applied Biosystems (Loughborough, UK), respectively.
Specificity test for primers and probes. The DNA of CAAresistant strain YQ1 and the CAA-sensitive strain LF1 were used as templates to test the specificities of the primers $(\mathrm{Cesa3F} /$ Cesa3R) and probes (Cesa3pR/Cesa3pS). To simulate the detection of the heterozygote genotype, $P$. viticola DNA of the CAAresistant strain was mixed with sensitive strain $L F 1$ in a 1:1 ratio. The fluorescence of Cesa3pR (FAM signal) was expected only from the resistant strain YQ1 and mixed DNA sample (YQ1-LF1), while the fluorescence of Cesa3pS (VIC signal) was expected only from sensitive strain and mixed DNA (YQ1-LF1). TaqMan-MGB realtime PCRs were performed in a $20 \mu \mathrm{l}$ volume containing $20 \mathrm{ng}$ of template DNA, $0.2 \mu \mathrm{M}$ of the forward and reverse primer, $1 \mu \mathrm{M}$ of Cesa3pR probe, $1 \mu \mathrm{M}$ of Cesa3pF probe, $10 \mu$ l of Probe qPCR Mix (Takara, Otsu, Japan), and finally $0.2 \mu l$ of ROX reference dye II was added as a passive internal reference. In each run, a negative amplification control (double distilled water, $\mathrm{ddH}_{2} \mathrm{O}$ ) was included. Amplifications were conducted in an ABI 7500 real-time PCR instrument (Applied Biosystems, Carlsbad, CA) programmed for $1 \mathrm{~min}$ at $60^{\circ} \mathrm{C}, 10 \mathrm{~min}$ at $95^{\circ} \mathrm{C}$, followed by 40 cycles of $15 \mathrm{~s}$ at $95^{\circ} \mathrm{C}$, and $1 \mathrm{~min}$ at $60^{\circ} \mathrm{C}$. The data were subsequently analyzed

Table 1. The detection of dimethomorph resistance in $P$. viticola isolates from main grape-growing areas in China by the TaqMan-minor groove binder (MGB) real-time PCR based method

\begin{tabular}{|c|c|c|c|c|c|c|}
\hline \multirow[b]{2}{*}{ Origin } & \multirow[b]{2}{*}{ No. of isolates } & \multirow[b]{2}{*}{ Resistant genotype isolate } & \multicolumn{2}{|c|}{ Sensitive genotype isolate } & \multirow[b]{2}{*}{ Resistance frequency $(\%)$} & \multirow{2}{*}{$\begin{array}{l}\text { Resistance allele } \\
\text { frequency }(\%)\end{array}$} \\
\hline & & & Homozygous & Heterozygous & & \\
\hline Beizhen, Liaoning & 327 & 316 & 8 & 3 & 96.6 & 97.1 \\
\hline Qingxu, Shanxi & 324 & 1 & 316 & 7 & 0.3 & 1.4 \\
\hline Wei, Hebei & 332 & 297 & 28 & 7 & 89.5 & 90.5 \\
\hline Penglai, Shandong & 315 & 278 & 30 & 7 & 88.3 & 89.4 \\
\hline Gongan, Hubei & 150 & 78 & 66 & 6 & 52.0 & 54.0 \\
\hline Huaihua, Hunan & 309 & 34 & 267 & 8 & 11.0 & 12.3 \\
\hline Binchuan, Yunnan & 216 & 19 & 192 & 5 & 8.8 & 10.0 \\
\hline Yongren, Yunnan & 254 & 164 & 88 & 2 & 64.6 & 65.0 \\
\hline Total & 2,227 & 1,187 & 995 & 45 & 53.3 & 54.3 \\
\hline
\end{tabular}

Table 2. Comparison among TaqMan-minor groove binder (MGB) real-time PCR, minimum inhibitory concentration (MIC), and sequencing method in evaluation of carboxylic acid amide (CAA) fungicide resistance in $P$. viticola isolates from Hebei Province

\begin{tabular}{|c|c|c|c|c|c|c|c|}
\hline Strain no. & MIC $^{\mathbf{a}}$ & TaqMan $^{b}$ & Sequencing ${ }^{c}$ & Strain no. & $\mathrm{MIC}^{\mathrm{a}}$ & TaqMan $^{b}$ & Sequencing ${ }^{c}$ \\
\hline $\mathrm{HeB} 1$ & $\mathrm{R}$ & $\mathrm{R}$ & $\mathrm{A} / \mathrm{A}$ & $\mathrm{HeB} 26$ & $S$ & $S$ & G/A \\
\hline $\mathrm{HeB} 2$ & S & $S$ & $\mathrm{G} / \mathrm{A}$ & $\mathrm{HeB} 27$ & $\mathrm{R}$ & $\mathrm{R}$ & $\mathrm{A} / \mathrm{A}$ \\
\hline $\mathrm{HeB} 3$ & S & S & $\mathrm{G} / \mathrm{G}$ & $\mathrm{HeB} 28$ & S & S & G/A \\
\hline $\mathrm{HeB} 4$ & $\mathrm{R}$ & $\mathrm{R}$ & $\mathrm{A} / \mathrm{A}$ & HeB29 & $\mathrm{R}$ & $\mathrm{R}$ & $\mathrm{A} / \mathrm{A}$ \\
\hline HeB5 & $\mathrm{R}$ & $\mathrm{R}$ & $\mathrm{A} / \mathrm{A}$ & HeB30 & $\mathrm{R}$ & $\mathrm{R}$ & $\mathrm{A} / \mathrm{A}$ \\
\hline HeB6 & $\mathrm{R}$ & $\mathrm{R}$ & $\mathrm{A} / \mathrm{A}$ & HeB31 & $\mathrm{R}$ & $\mathrm{R}$ & $\mathrm{A} / \mathrm{A}$ \\
\hline $\mathrm{HeB} 7$ & $\mathrm{R}$ & $\mathrm{R}$ & $\mathrm{A} / \mathrm{A}$ & HeB32 & S & S & $\mathrm{G} / \mathrm{G}$ \\
\hline $\mathrm{HeB} 8$ & $\mathrm{R}$ & $\mathrm{R}$ & $\mathrm{A} / \mathrm{A}$ & HeB33 & $\mathrm{R}$ & $\mathrm{R}$ & $\mathrm{A} / \mathrm{A}$ \\
\hline HeB9 & S & $S$ & $\mathrm{G} / \mathrm{G}$ & HeB34 & $\mathrm{R}$ & $\mathrm{R}$ & $\mathrm{A} / \mathrm{A}$ \\
\hline $\mathrm{HeB} 10$ & S & $S$ & $\mathrm{G} / \mathrm{G}$ & HeB35 & $\mathrm{R}$ & $\mathrm{R}$ & $\mathrm{A} / \mathrm{A}$ \\
\hline $\mathrm{HeB} 11$ & $\mathrm{R}$ & $\mathrm{R}$ & $\mathrm{A} / \mathrm{A}$ & HeB36 & S & S & $\mathrm{G} / \mathrm{G}$ \\
\hline $\mathrm{HeB} 12$ & $\mathrm{R}$ & $\mathrm{R}$ & $\mathrm{A} / \mathrm{A}$ & HeB37 & $S$ & S & $\mathrm{G} / \mathrm{G}$ \\
\hline HeB13 & $\mathrm{R}$ & $\mathrm{R}$ & $\mathrm{A} / \mathrm{A}$ & HeB38 & S & S & $\mathrm{G} / \mathrm{G}$ \\
\hline $\mathrm{HeB} 14$ & $\mathrm{R}$ & $\mathrm{R}$ & $\mathrm{A} / \mathrm{A}$ & HeB39 & $\mathrm{R}$ & $\mathrm{R}$ & $\mathrm{A} / \mathrm{A}$ \\
\hline HeB15 & S & $S$ & $\mathrm{G} / \mathrm{G}$ & $\mathrm{HeB} 40$ & $\mathrm{R}$ & $\mathrm{R}$ & $\mathrm{A} / \mathrm{A}$ \\
\hline $\mathrm{HeB} 16$ & $\mathrm{R}$ & $\mathrm{R}$ & $\mathrm{A} / \mathrm{A}$ & $\mathrm{HeB} 41$ & $\mathrm{R}$ & $\mathrm{R}$ & $\mathrm{A} / \mathrm{A}$ \\
\hline $\mathrm{HeB} 17$ & $\mathrm{R}$ & $\mathrm{R}$ & $\mathrm{A} / \mathrm{A}$ & $\mathrm{HeB} 42$ & $\mathrm{R}$ & $\mathrm{R}$ & $\mathrm{A} / \mathrm{A}$ \\
\hline HeB18 & S & $S$ & $\mathrm{G} / \mathrm{G}$ & $\mathrm{HeB} 43$ & $S$ & S & $\mathrm{G} / \mathrm{G}$ \\
\hline HeB19 & $S$ & $S$ & $\mathrm{G} / \mathrm{G}$ & HeB44 & S & S & $\mathrm{G} / \mathrm{G}$ \\
\hline $\mathrm{HeB} 20$ & $\mathrm{R}$ & $\mathrm{R}$ & $\mathrm{A} / \mathrm{A}$ & $\mathrm{HeB} 45$ & $\mathrm{R}$ & $\mathrm{R}$ & $\mathrm{A} / \mathrm{A}$ \\
\hline $\mathrm{HeB} 21$ & $\mathrm{R}$ & $\mathrm{R}$ & $\mathrm{A} / \mathrm{A}$ & HeB46 & $\mathrm{R}$ & $\mathrm{R}$ & $\mathrm{A} / \mathrm{A}$ \\
\hline $\mathrm{HeB} 22$ & S & S & G/A & HeB47 & $\mathrm{R}$ & $\mathrm{R}$ & $\mathrm{A} / \mathrm{A}$ \\
\hline $\mathrm{HeB} 23$ & $\mathrm{R}$ & $\mathrm{R}$ & $\mathrm{A} / \mathrm{A}$ & $\mathrm{HeB} 48$ & $\mathrm{R}$ & $\mathrm{R}$ & $\mathrm{A} / \mathrm{A}$ \\
\hline $\mathrm{HeB} 24$ & $\mathrm{R}$ & $\mathrm{R}$ & $\mathrm{A} / \mathrm{A}$ & HeB49 & $S$ & S & $\mathrm{G} / \mathrm{G}$ \\
\hline $\mathrm{HeB} 25$ & S & S & $\mathrm{G} / \mathrm{G}$ & HeB50 & $\mathrm{R}$ & $\mathrm{R}$ & $\mathrm{A} / \mathrm{A}$ \\
\hline
\end{tabular}

${ }^{\mathrm{a}} \mathrm{MIC}>1.6 \mu \mathrm{g} \mathrm{ml}^{-1}$ was regarded as a resistant isolate (R), and $\mathrm{MIC}<1.6 \mu \mathrm{g} \mathrm{ml}{ }^{-1}$ was regarded as a sensitive isolate (S) (Sun et al. 2010).

${ }^{\mathrm{b}} \mathrm{S}$ and $\mathrm{R}$ represent sensitive and resistant genotypes identified by TaqMan-MGB.

${ }^{c} \mathrm{G} / \mathrm{G}, \mathrm{A} / \mathrm{A}$, and G/A represent sensitive homozygote, resistant homozygote, and sensitive heterozygote genotypes at the position in the $P v C e s A 3$ genes identified by sequencing. 
with 7500 Software Version 2.0.6 (Applied Biosystems, Carlsbad, CA).

Sensitivity of the new method. The sensitivity of the TaqManMGB RT-PCR based method was determined using a 10-fold serial dilution of total DNA over a five-log scale. For YQ1, $0.126 \mathrm{pg}$ to $12.6 \mathrm{ng}$; for LF1, $0.205 \mathrm{pg}$ to $20.5 \mathrm{ng}$; and for the 1:1 mixture of the both strains, $5 \mathrm{pg}$ to $50 \mathrm{ng}$ was used as the template. The correlated cycle threshold $\left(C_{T}\right)$ values were used to set the standard curves for respective absolute quantifications (Decaro et al. 2005). The double distilled water was used as negative amplification control. The tests were performed in three replicates.

Leaf disc assay. The conventional leaf disc method was used to evaluate the dimethomorph resistance of $P$. viticola (Wong and Wilcox 2000). Briefly, 50 purified strains from Wei County were inoculated on the leaf discs using the method described above. Seven days later, the sporangia and sporangiophores were collected in a tube with sterile water. After the suspension was filtered through a $30-\mu \mathrm{m}$ sterile mesh, the sporangia were counted under a microscope (BX41, Olympus, Tokyo, Japan) using a hemocytometer and adjusted to $10^{4}$ sporangia $\mathrm{ml}^{-1}$ to serve as the $P$. viticola inoculum. The dimethomorph ( $97 \%$ active ingredient) used in this study was kindly provided by the pesticide laboratory of the Institute of Plant Protection, Chinese Academy of Agricultural Sciences, Beijing, China. According to the report of Sun et al. (2010), the fungicide was dissolved in methanol and diluted with water to a concentration of $1.6 \mu \mathrm{g} \mathrm{ml}^{-1}$. Fully expanded leaves of $V$. vinifera 'Rizamat,' four to six leaf positions distal from the shoot tip, were washed with tap water thoroughly, surface disinfested for $90 \mathrm{~s}$ in sterile water containing $0.5 \%$ sodium hypochlorite and $0.1 \%$ Tween 20 (Hushi, Shanghai, China), triple rinsed in sterile water, and blotted dry between paper towels. Discs (diameter $15 \mathrm{~mm}$ ) from leaves were incubated for $1 \mathrm{~h}$ in the fungicide solution and transferred onto rifampicinampicillin-pimaricin (RAP) agar medium (Wong and Wilcox 2000; Zhang et al. 2017). Ten discs per plate were used for each isolate and the abaxial side of each disc was inoculated with a 10- $\mu$ l drop of a $P$. viticola suspension. The dimethomorph resistant isolate YQ1 and the sensitive isolate LF1 were used as positive and negative controls, respectively. Leaf discs treated with a solution without dimethomorph were used as a blank control. After 8 days of incubation at $21^{\circ} \mathrm{C}$ and a 16 -h photoperiod, downy mildew symptoms on each leaf disc were recorded. If the symptoms were present at the minimum inhibitory concentration (MIC, $1.6 \mu \mathrm{g} \mathrm{ml}^{-1}$ ) in the treated discs of experimental and positive control groups, and not in the negative and blank controls, the isolate was regarded as resistant; if they infected the discs of the positive control, but not the experimental, negative, and blank control groups, isolates were designated as sensitive (Sun et al. 2010). The test was repeated three times.

Sequence analysis. DNA sequencing of part of the PvCesA3 gene of $50 P$. viticola isolates was conducted to confirm the point mutations at codon 1105 of isolates in the present study. PCR primers for DNA sequencing, PVcesa3F and PVcesa3R, were designed to amplify a $603 \mathrm{bp}$ fragment including codon 1105 using the software Primer Premier 5.0 (PREMIER Biosoft, PaloAlto, CA). Primer information is shown in Table 3. PCR amplification was performed in a total volume of $20 \mu \mathrm{l}$ including $20 \mathrm{ng}$ of $P$. viticola DNA template, $0.5 \mu \mathrm{M}$ of each primer, $10 \mathrm{mM}$ of Tris- $\mathrm{HCl}$ (pH 8.3), $50 \mathrm{mM}$ of $\mathrm{KCl}$, $1.5 \mathrm{mM}$ of $\mathrm{MgCl}_{2}, 250 \mu \mathrm{M}$ of each dNTP, and 0.5 units of Taq polymerase (Tiangen, Beijing, China). PCR amplification was performed in a Veriti 96-well thermal cycler (Applied Biosystems, Carlsbad, CA) with the following temperature profile: an initial denaturation at $95^{\circ} \mathrm{C}$ for $5 \mathrm{~min}$; followed by 35 cycles of denaturation at $95^{\circ} \mathrm{C}$ for $30 \mathrm{~s}$, annealing at $57^{\circ} \mathrm{C}$ for $30 \mathrm{~s}$, and extension at $72^{\circ} \mathrm{C}$ for $30 \mathrm{~s}$; and a final extension at $72^{\circ} \mathrm{C}$ for $10 \mathrm{~min}$. PCR products were sequenced using Sanger sequencing by a Sangon Biotech Company sequencer (Shanghai, China). Subsequently, the partial PvCesA3 sequences were examined and aligned with other reference sequences from GenBank database (GQ258975.1, KU860534.1 542.1) using Geneious 11.1.5 (Biomatters, Ltd., Auckland, New Zealand).

Monitoring of dimethomorph resistance of $P$. viticola. To understand the frequency of CAA fungicide resistance in $P$. viticola samples in China, the genotype of PvCesA3 of 2,227 P. viticola isolates (Table 1) obtained from vineyards located in the eight main grapevine growing areas (Fig. 1) were detected by the TaqManMGB real-time PCR based method.

Two or three large vineyards were selected as representatives in each sampled region where the dimethomorph treatment including frequency of applications and total amounts used were surveyed from 2014 to 2016 (Supplementary Table S1). The correlation between the total frequency of CAA-resistant isolates and annual average dimethomorph dosage and average fungicide application frequency,

Table 3. Primers and probes used in this study

\begin{tabular}{|c|c|c|}
\hline Name & Sequence $\left(5^{\prime}-3^{\prime}\right)$ & $\begin{array}{l}\text { Product length } \\
\text { (bp) }\end{array}$ \\
\hline Cesa3pR ${ }^{a}$ & FAM-AGCAACGAGCTGAA-MGB & \\
\hline Cesa3pS a & VIC-CAGCAACGAGCCGA-MGB & \\
\hline Cesa3F $F^{b}$ & CGGCTGCTACCTTTACGGCAAA & 66 \\
\hline Cesa3R ${ }^{b}$ & GCACAAACACGACAATGTAGACAA & \\
\hline PVcesa3F $\mathrm{F}^{\mathrm{c}}$ & CCGATTTACGCTCGTGGTACCA & 603 \\
\hline $\mathrm{PV} \operatorname{ces} 33 \mathrm{R}^{\mathrm{c}}$ & CTACGTCACAGTCGTGGCAGCA & \\
\hline \multicolumn{3}{|c|}{$\begin{array}{l}\text { a The specific TaqMan-minor groove binder (MGB) probes for resistant } \\
\text { (Cesa3pR) and sensitive allele (Cesa3pS), respectively. FAM (carboxy- } \\
\text { fluorescein) and VIC (Applied Biosystems, Carlsbad, CA) at the 5' end rep- } \\
\text { resent the reporter dye; MGB at the } 3^{\prime} \text { end represents a nonfluorescent } \\
\text { quencher (NFQ). } \\
\text { b The specific primer for TaqMan-MGB real-time PCR. } \\
\text { c PCR primers for DNA sequencing. }\end{array}$} \\
\hline
\end{tabular}

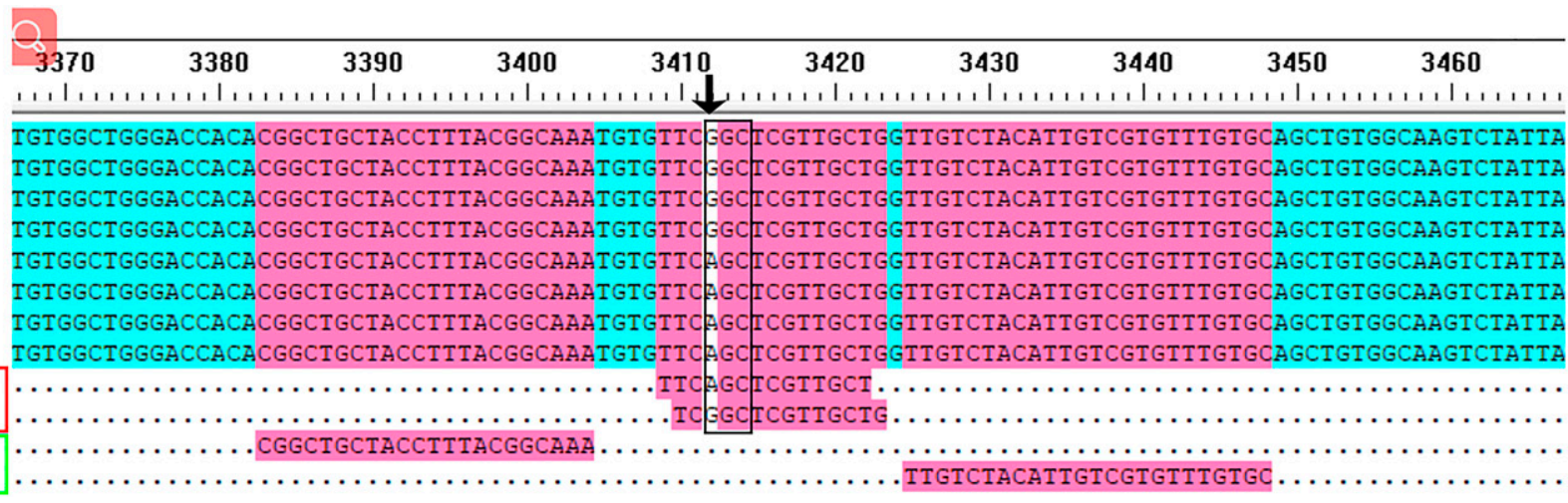

Fig. 2. Minor groove binder (MGB) probe and primer map for TaqMan-MGB real-time PCR analysis of $P v C e s A 3$. They were designed from $P v C e s A 3$ of $P$. viticola (complete cds: accession no. GQ258975 and partial cds: accession nos. KU860541.1, KU860539.1, KU860536.1, KU860542.1, KU860540.1, KU860535.1, and KU860534.1). The black arrow and black box respectively indicate the point mutation and codon 1105 of the PvCesA3 gene conferring carboxylic acid amide (CAA) fungicide resistance, of which GGC (Gly) represents the sensitive allele, AGC (Ser) represents the resistant allele. The red box indicates the probes (Cesa3pS: positions 3409-3422; Cesa3pR: positions 3410-3423); the green box indicates the primer (Cesa3F: positions 3383-3404; Cesa3R: positions 3425-3448). 
respectively, in each sample region was determined using the generalized linear model (GLM) and Spearman's correlation coefficient, which were performed in the software package $\mathrm{R}$ (version 3.6.1) with glm and cor function.

\section{Results}

Establishment of a TaqMan-MGB real-time PCR based method. Specificity of primers and probes. Using the DNA of resistant (YQ1) and sensitive (LF1) isolates and their mixture, the specificity of the TaqMan-
MGB probes and primers were tested. The results showed that the FAM signals were detected only when a sample contained the $P v C e s A 3$ resistant allele (YQ1 and the mixture of YQ1 and LF1). VIC signals were detected only when a sample contained DNA with the sensitive allele (Fig. 3). The allelic discrimination of YQ1 and LF1 was in agreement with the leaf disc test and DNA sequencing and this new method can detect heteroallelic $P v C e s A 3$ isolates.

Efficiency and sensitivity. The efficiency and sensitivity of each assay was determined using the amplification plots and standard

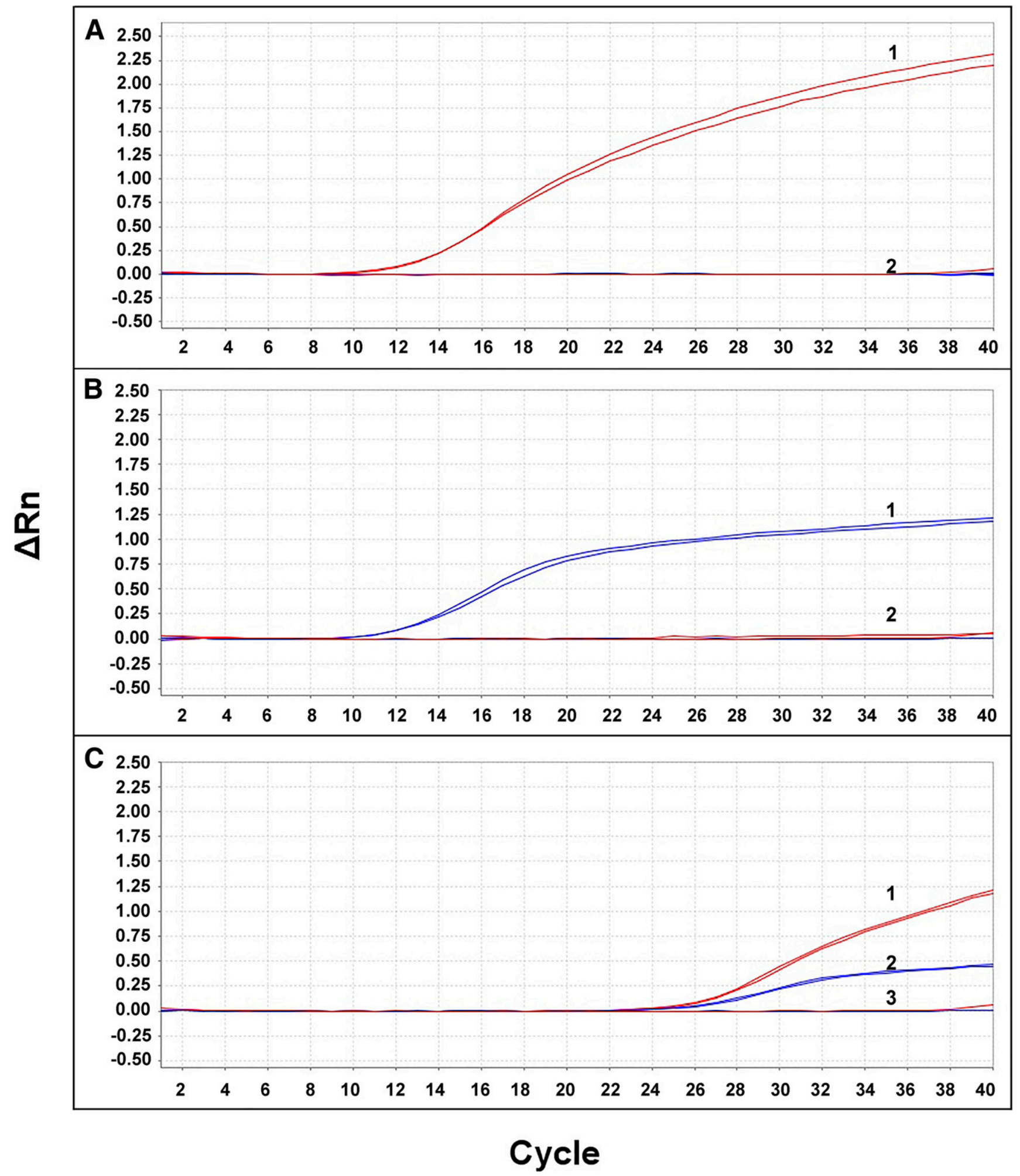

Fig. 3. Specificity of the primer pair and TaqMan probes of TaqMan-minor groove binder (MGB) real-time PCR. A, Amplification of the DNA from the dimethomorph-resistant isolate YQ1 (curve 1: resistant allele; curve 2: negative control). B, Amplification of the DNA from the dimethomorph-sensitive isolate LF1 (curve 1: sensitive allele; curve 2: negative control). C, Amplification of the mix DNA of YQ1 and LF1 (curve 1: resistant allele; curve 2: sensitive allele; curve 3: negative control). 
curves. As shown by the results in Figure 4, the slopes of these assays were between -3.562 and -3.415 (90.9-96.3\% efficiency), with a correlation coefficient exceeding 0.99 (Fig. 4B, D, F). For the resistant strain, TaqMan-MGB RT-PCR detected the resistant genotype in all three replicates from $0.126 \mathrm{pg}$ to $12.6 \mathrm{ng}$. No signal was obtained with the sensitive allele, even at $12.6 \mathrm{ng}$ (Fig. 4A). The detection limit was determined at $0.126 \mathrm{pg}$ and the specificity for target versus nontarget of $>10,000$-fold. The sensitive allele was detected in all three replicates from $0.205 \mathrm{pg}$ to $20.5 \mathrm{ng}$. No signal was observed for the resistant allele, even at $20.5 \mathrm{ng}$ (Fig. 4C). This indicates a detection limit of $0.205 \mathrm{pg}$ and a specificity for target versus nontarget of $>10,000$-fold. For the mixture DNA of the two strains, the resistant and sensitive alleles were simultaneously detected from $5 \mathrm{pg}$ to 50 ng (Fig. 4E). In all the reactions, the negative PCR control did not generate any signal (Fig. 4).

Verification of the TaqMan-MGB real-time PCR based method. The TaqMan-MGB real-time PCR method was validated by subjecting 50 single-sporangiophore isolates to the leaf disc assay and Sanger sequence analysis of partial $P v C e s A 3$ gene. The TaqMan-MGB real-time PCR identified 32 isolates with the CAA-resistant genotype, which were homozygous for the resistant allele of $P v C e s A 3$. The other 16 isolates showed the CAA-sensitive genotype; 14 of them were homozygous for the sensitive allele and four were heterozygotes including both the sensitive and resistant allele. The genotypes determined by the TaqMan-MGB real-time PCR were in accordance with the DNA sequencing of the mutated positions in PvCesA3 genes (Table 2). All homozygous resistant isolates, as determined by molecular methods, infected the leaf disks in the in vivo bioassay, even when $1.6 \mu \mathrm{g} \mathrm{ml}^{-1}$ dimethomorph was applied. In contrast, dimethomorph completely inhibited infection by the sensitive genotype isolates, including both the homozygous and heterozygotes (Table 2). This verified that the present TaqMan-MGB realtime PCR based method can specifically detect the resistant and sensitive alleles of $P v C e s A 3$ gene and assess the CAA fungicide resistance found in $P$. viticola populations.

Detection of dimethomorph resistant and sensitive genotypes of $\boldsymbol{P}$. viticola in China. The TaqMan-MGB real-time PCR was used to survey the frequency of the dimethomorph-resistant $P$. viticola isolates from a collection of 2,227 P. viticola single-sporangiophore
A
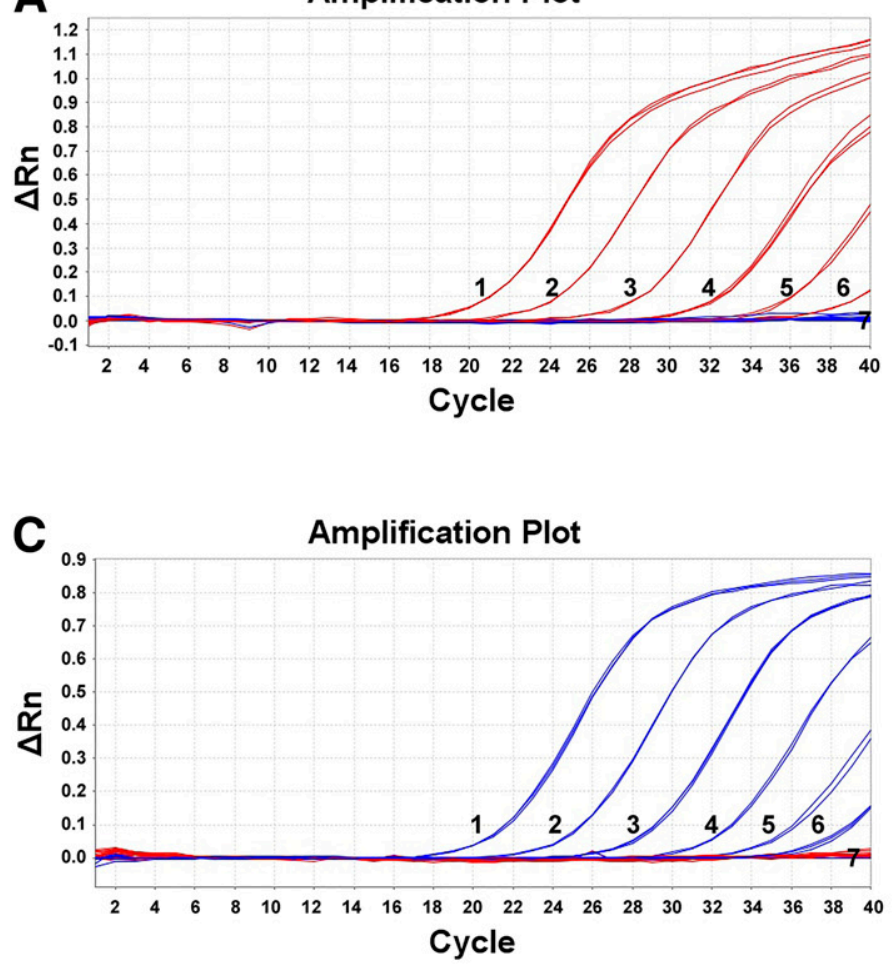

E

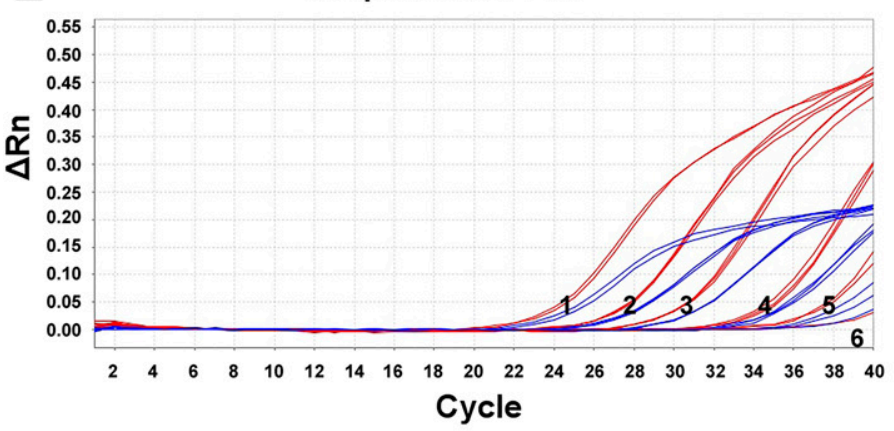


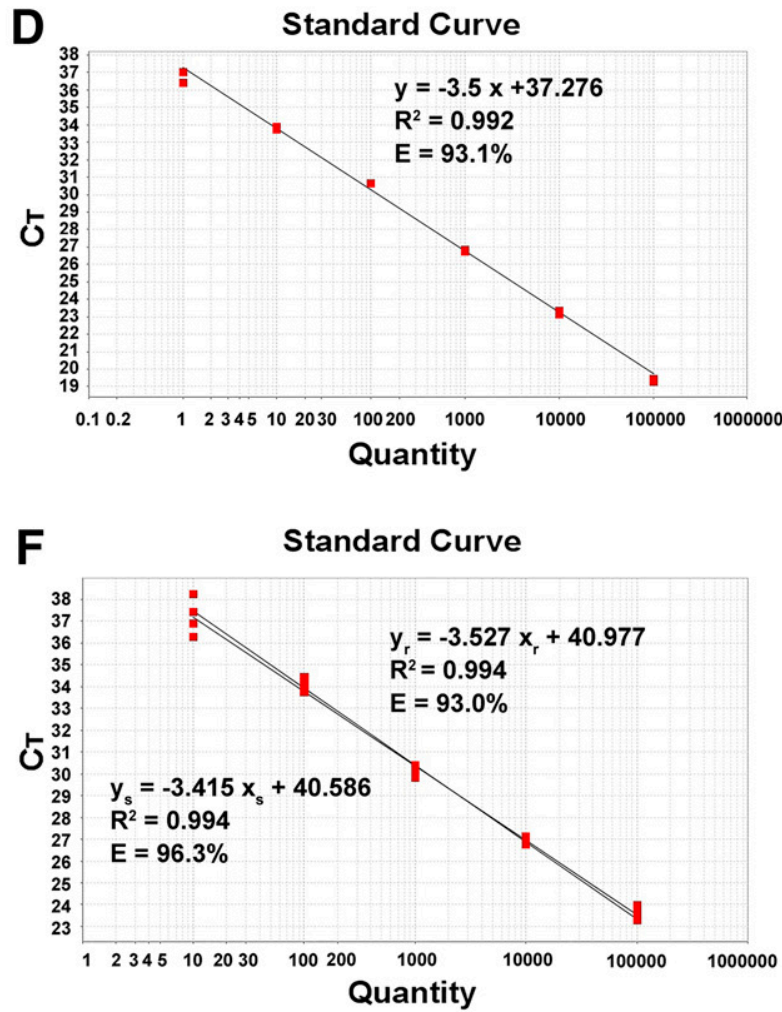

Fig. 4. Amplification plots and standard curves of the TaqMan-minor groove binder (MGB) real-time PCR based method with 10-fold dilutions of DNA from resistance allele (A and B) of the PvCesA3 gene (lines 1-6: 12.6 ng to 0.126 pg; line 7: negative control); or sensitive allele (C and D) of the PvCesA3 gene (lines 1-6: 20.5 ng to 0.205 pg; line 7: negative control) or mixture of the resistant and sensitive allele (E and F) of the PvCesA3 gene (lines 1-5: $50 \mathrm{ng}$ to $5 \mathrm{pg}$; line 6: negative control). For all the amplification plots, the red lines represent the resistant allele, and blue lines represent the sensitive allele. 
isolations originating from eight locations in China (Table 1). The CAA-resistant genotype was present in 1,187 isolates (53.3\%), and 1,040 isolates showed the genotype sensitive to CAA fungicide, of which 45 were heterozygotes. The frequencies of resistance from high to low was Beizhen (96.6\%), Wei County (89.5\%), Penglai (88.3\%), Yongren (64.6\%), Gongan (52\%), Binchuan (8.8\%), and Qingxu (0.3\%) (Fig. 1). In addition, the frequencies of resistant alleles were higher than the frequencies of resistance genotypes in all the regions, but the trend was consistent (Table 1). Furthermore, the total resistant genotype frequency in each region was significantly correlated with the local average fungicide dosage (Spearman's correlation: rho $=0.90, P=0.0045$; GLM: $P=0.0295)$ and average application frequency (Spearman's correlation: rho $=0.95$, $P=0.0011$; GLM: $P=0.0009$ ), respectively (Supplementary Fig. S1).

\section{Discussion}

In this study, TaqMan RT-PCR primers and MGB probes specific to allele G1105S that conferred resistance of $P$. viticola to dimethomorph were successfully designed, validated, and applied. Compared with the conventional leaf disc method, the molecular methods used to detect fungicide resistance are much quicker (Miao et al. 2016). Therefore, a number of PCR-based methods had been developed to monitor the shift in the CAA fungicide sensitivity of $P$. viticola field populations (Aoki et al. 2011, 2013; Zhang et al. 2017). Among them, the multiplex allele-specific primer (ARMS) PCR assay was developed to simultaneously detect QoI and CAA fungicide resistance alleles in P. viticola (Aoki et al. 2013). This method was simple and rapid, but it can just detect the existence of the resistant allele and is unable to distinguish the homo- and heterozygote genotype of PvCesA3. Previously, we developed a tetra-primer ARMS PCR to detect the CAA-resistant $P$. viticola (Zhang et al. 2017). It not only detected the resistant alleles, but also could discriminate between homo- and heterozygote genotypes. However, the presence of two pairs of primers in one reaction might result in cross-amplification and false positive results (Hamajima et al. 2002), which was also observed in our experiments (Zhang et al., unpublished data). Due to significantly improved hybridization properties of MGB probes (Kutyavin et al. 2000), we opted to employ TaqMan-MGB probes, allowing a shorter probe and higher specificity of the probe (Ma et al. 2013). The use of TaqMan-MGB real-time PCR enabled specific distinction of resistant and sensitive genotypes of $P$. viticola to dimethomorph and other CAAs and enhanced specificity, sensitivity, and throughput.

Two-point mutations at the same codon (G1105S and G1105V) have been reported to result in CAA resistance (Blum et al. 2010b; Sierotzki et al. 2011). The G1105S mutation was predominant in most areas of Europe (Blum et al. 2010b; Sierotzki et al. 2011) and Japan (Aoki et al. 2015). In our previous study we also only detected this genotype in Guangxi Province in China (Zhang et al. 2017). So far, the G1105V mutation is only detected in a few locations worldwide and at low frequency (reviewed by Zhang et al. 2017). Therefore, the resistant frequency based on identification of G1105S mutation is currently sufficient to assess CAA fungicide resistance in China. Nevertheless, we suggest that in addition to the genotypic surveys, phenotypic surveys should also be performed to monitor other possible mutations and to establish a living collection that can be stored at $-20^{\circ} \mathrm{C}$ (Delmotte et al. 2014). This will help to gain a more comprehensive understanding of mutations conferring CAA resistance of $P$. viticola in China.

As $P$. viticola is an obligate biotrophic pathogen, genotyping is performed in the background of the host and low concentrations of $P$. viticola DNA from samples are expected. The TaqMan-MGB real-time PCR assay developed in this study could detect the resistant homozygous allele in $0.126 \mathrm{pg}$ total DNA and homozygous sensitive allele in $0.205 \mathrm{pg}$ total DNA; the heterozygote genotype could be detected in 5 pg DNA. The sensitivity was much higher than the RFLP PCR (2 ng) (Aoki et al. 2011). Subsequently, Aoki et al. (2013) developed a multiplex allele-specific primer PCR assay (ARMS), which greatly improved the detection sensitivity, but could not distinguish between homo- and heterozygote genotype required for the correct identification of resistant isolates. The high sensitivity was further exploited to use the assay on infected leaf discs. Using the TaqMan-MGB real-time PCR assay, the homozygote and heterozygote genotypes of the mutation sites (G1105S) in PvCesA3 gene conferring CAA resistance were successfully detected in the DNA of infected leaf discs of the 50 isolates from Wei County. The results were in agreement with the direct sequencing and fungicide sensitivity assays in vitro, suggesting that the new method is robust and reliable for infected leaf disc samples in the laboratory. This would omit the step of isolation of the isolates and further enhance the throughput. However, we did not test the specificity of this method; therefore, further research is needed for the potential application of direct screening of infected leaf samples from the field, which would allow identification of CAA fungicide resistance in a high density grid required to assess spatial and temporal distributions and assist decision support during the season.

Dimethomorph has been widely applied in Chinese vineyards since it was registered in 1996 for the control of $P$. viticola (Sun et al. 2010). In 2010, Sun et al. tested the sensitivity of 392 P. viticola isolates collected from 11 regions in seven provinces in China by the conventional leaf disk assay and no resistant isolates were detected (Sun et al. 2010). In 2014, dimethomorph-resistant isolates of $P$. viticola were first collected in Guangxi Province, China (Zhang et al. 2017). In this study, we investigate the CAA-fungicide resistance frequency of a large $P$. viticola population from eight main grapegrowing areas in China. The resistance frequency is significantly correlated with the use of the CAA fungicides $(P<0.05)$. Beizhen, Penglai, and Wei (resistant frequency $>88 \%$ ) are all traditional grape-growing areas since the 1950s and in these regions, CAA fungicides have been applied over a prolonged period of time. In the period from 2014 to 2016, the use of dimethomorph reached roughly $2.38 \mathrm{~kg}$ per hectare on average in those regions; the number of CAA treatments in those regions ranged from three to seven (Supplementary Table S1), and the average fungicide use frequency exceeded the maximum number recommended by FRAC. Those might result in a strong selection for a resistant population. This is in contrast to the situation in Qingxu, which is also a traditional grape growing region located in northern China; due to its relatively low level of economic development, the application of CAA fungicides was much lower (about $0.06 \mathrm{~kg} / \mathrm{ha}$; most winegrowers applied one time; Supplementary Table S1), and mainly copper products have been used to control grape downy mildew. This markedly lower selection pressure resulted in the lowest resistance frequency $(0.3 \%)$, indicating dimethomorph is still an effective fungicide for grape downy mildew control in Qingxu. The CAA resistance frequency of Gongan, Yongren, Binchuan, and Huaihua ranged from 8.8 to $64.6 \%$, being significantly lower than Beizhen, Penglai, or Wei $(>88 \%)$. These regions are located in southern China, where the humidity is high and the climate is conducive for grape downy mildew; therefore, the application of rain shelter cultivation is now popular, and this greatly reduced this disease as well as the dosage and application frequency of fungicides. The average dosage $(1.09 \mathrm{~kg} / \mathrm{ha})$ and application frequency $(3.2$ times) in those regions are both significantly $(P<0.001, t$ test $)$ less than in northern regions (2.38 kg/ha, 5.1 times) from 2014 to 2016 (Supplementary Table S1). In addition, those regions were new grape-growing areas since the early 1990s. The short-term and lower frequency and dosage of fungicide application may result in lower resistance frequencies of $P$. viticola. Finally of interest is the difference between Binchuan and Yongren, as they are located nearby and the climate is very similar; however, the CAA resistance frequencies in these regions are significantly different. This may be due to the more complex and larger variation in the fungicide application in Binchuan. This could result in a lower selective pressure of CAA fungicides and lower frequencies of resistance. In the regions with lower frequencies of CAA resistance, it is strongly recommended that CAA fungicides should be applied to control grape downy mildew disease according to FRAC recommendations (FRAC 2017), which include application of CAA fungicides preferably in a preventive manner (applying before the pathogen arrives or begins to develop), 
and limiting the total number of CAA fungicide sprays to less than four per crop cycle. In the areas with high CAA resistance frequency, strategies should be implemented to prevent or decrease the further spread of resistant fungal subpopulations in the field, such as planting more resistant cultivars and applying CAA fungicides in a mixture with effective fungicides from other classes.

In addition, $4.3 \%$ of the sensitive strains were heterozygous, resulting in a higher resistance allele frequency. The resistance allele frequency may increase due to asexual spread of resistant strains as well as due to crossings in which the progeny segregates for the resistance allele and selection of resistant progeny. Limited information is available on the sexual cycle of $P$. viticola in the field in China. Yin et al. investigated the population diversity of a nationwide collection of $P$. viticola in China by SSR markers. They found high genotypic diversity in most regions and concluded that sexual reproduction contributes significantly to epidemics of $P$. viticola (Yin et al. 2014). However, linkage equilibrium among these loci was not analyzed, making it hard to evaluate the level of sexual reproduction. In this study, a low ratio of heterozygotes was found in all regions (0.79 to $4 \%$ ); especially in Gongan and Yongren, the frequency of heterozygotes is much lower than expected based on the allele frequencies $(P<0.0001)$. The lower fitness of the heterozygote when exposed to CAA fungicides could result in elimination of heterozygous progeny after the sexual cycle. Alternatively, the bias could be due to the asexual spread of resistant genotypes. Therefore, more population diversity and fitness studies are needed to identify the main drivers of the increase of resistance allele frequency.

In brief, we describe an easy and reliable TaqMan-MGB RT-PCR based method that could directly evaluate CAA resistance from infected plant tissue and simultaneously detect the resistant homozygote, the sensitive heterozygote, and the sensitive homozygote. Based on this method, CAA fungicide resistance frequency of a comprehensive collection of $P$. viticola from main grape-growing areas in China was monitored. To our knowledge, this collection of $P$. viticola isolates is the largest in China. The size and the sampling strategy in one season allowed detailed analyses of CAA fungicide resistance. The results provide important information on the resistant frequency of $P$. viticola to CAA fungicides in China and will improve the management of grape downy mildew in the future.

\section{Acknowledgment}

We thank Cees Waalwijk for critically reading the manuscript.

\section{Literature Cited}

Aoki, Y., Furuya, S., and Suzuki, S. 2011. Method for rapid detection of the $P v C e s A 3$ gene allele conferring resistance to mandipropamid, a carboxylic acid amide fungicide, in Plasmopara viticola populations. Pest Manag. Sci. 67:1557-1561

Aoki, Y., Hada, Y., and Suzuki, S. 2013. Development of a multiplex allelespecific primer PCR assay for simultaneous detection of QoI and CAA fungicide resistance alleles in Plasmopara viticola populations. Pest Manag. Sci. 69:268-273.

Aoki, Y., Kawagoe, Y., Fujimori, N., Tanaka, S., and Suzuki, S. 2015. Monitoring of a single point mutation in the $P v C e s A 3$ allele conferring resistance to carboxylic acid amide fungicides in Plasmopara viticola populations in Yamanashi Prefecture, Japan. Plant Health Prog. 16:84-87.

Blum, M., Boehler, M., Randall, E., Young, V., Csukai, M., Kraus, S., Moulin, F., Scalliet, G., Avrova, A. O., Whisson, S. C., and Fonne-Pfister, R. 2010a. Mandipropamid targets the cellulose synthase-like PiCesA3 to inhibit cell wall biosynthesis in the oomycete plant pathogen, Phytophthora infestans. Mol. Plant Pathol. 11:227-243.

Blum, M., Waldner, M., and Gisi, U. 2010b. A single point mutation in the novel $P v C e s A 3$ gene confers resistance to the carboxylic acid amide fungicide mandipropamid in Plasmopara viticola. Fungal Genet. Biol. 47:499-510.

Chen, W. J., Delmotte, F., Richard-Cervera, S., Douence, L., Greif, C., and CorioCostet, M. F. 2007. At least two origins of fungicide resistance in grapevine downy mildew populations. Appl. Environ. Microbiol. 73:5162-5172.

Decaro, N., Martella, V., Ricci, D., Elia, G., Desario, C., Campolo, M., Cavaliere, N., Di Trani, L., Tempesta, M., and Buonavoglia, C. 2005. Genotype specific fluorogenic
RT PCR assays for the detection and quantitation of canine coronavirus type I and type II RNA in faecal samples of dogs. J. Virol. Methods 130:72-78.

Emmett, R. W., Wicks, T. J., and Magarey, P. A. 1992. Downy mildew of grapes. Pages 90-128 in: Plant Diseases of International Importance. Vol II: Diseases of Fruit Crops. J. Kumar, H. S. Chaube, U. S. Singh, and A. N. Mukhopadhyay, eds. Prentice Hall, Englewood Cliffs, NJ, U.S.A.

Fan, J., Luo, Y., Michailides, T. J., and Guo, L. 2014. Simultaneous quantification of alleles E198A and H6Y in the $\beta$-tubulin gene conferring benzimidazole resistance in Monilinia fructicola using a duplex real-time (TaqMan) PCR. Pest Manag. Sci. 70:245-251.

FRAC. 2017. Protocol of the discussions and recommendations of the CAA Working Group of the Fungicide Resistance Action Committee (FRAC). Available: https:// www.frac.info/docs/default-source/working-groups/caa-fungicides/caa-wg/ minutes-of-the-2017-caa-meeting-recommendations-for-2018.pdf.

Gisi, U., Lamberth, C., Mehl, A., and Seitz, T. 2007a. Carboxylic acid amide (CAA) fungicides. Pages 651-674 in: Modern Crop Protection Compounds. W. Krämer and U. Schirmer, eds. Wiley-VCH, Weinheim, Germany.

Gisi, U., and Sierotzki, H. 2008. Fungicide modes of action and resistance in downy mildews. Eur. J. Plant Pathol. 122:157-167.

Gisi, U., Waldner, M., Kraus, N., Dubuis, P. H., and Sierotzki, H. 2007b. Inheritance of resistance to carboxylic acid amide (CAA) fungicides in Plasmopara viticola. Plant Pathol. 56:199-208.

Hamajima, N., Saito, T., Matsuo, K., and Tajima, K. 2002. Competitive amplification and unspecific amplification in polymerase chain reaction with confronting two-pair primers. J. Mol. Diagn. 4:103-107.

Hu, P., Li, X. H., Zhang, X. L., Geng, W. L., Cai, X. N., Liu, Z. P., and Wei, Y. M 2013. A field survey and control efficacy test of grape downy mildew. Chin. Agr. Sci. Bull. 29:181-185.

Kutyavin, I., Afonina, I. A., Mills, A., and Hedgpeth, J. 2000. 3'-Minor groove binder-DNA probes increase sequence specificity at PCR extension temperatures. Nucleic Acids Res. 28:655-661.

Ma, M., Liu, J., Song, Y., Li, L., and Li, Y. 2013. TaqMan MGB probe fluorescence real-time quantitative PCR for rapid detection of Chinese Sacbrood virus. PLoS One 8:e52670.

Miao, J. Q., Dong, X., Lin, D., Wang, Q. S., Liu, P. F., Chen, F. R., Du, Y. X., and Liu, X. L. 2016. Activity of the novel fungicide oxathiapiprolin against plantpathogenic oomycetes. Pest Manag. Sci. 72:1572-1577.

Myles, S., Boyko, A. R., Owens, C. L., Brown, P. J., Grassi, F., Aradhya, M. K., Prins, B., Reynolds, A., Chia, J. M., Ware, D., Bustamante, C. D., and Buckler, E. S. 2011. Genetic structure and domestication history of the grape. Proc. Natl. Acad. Sci. USA 108:3530-3535.

OIV. 2018. OIV Statistical Report on World Vitiviniculture. http://oiv.int/public/ medias/6371/oiv-statistical-report-on-world-vitiviniculture-2018.

REX Consortium. 2013. Heterogeneity of selection and the evolution of resistance. Trends Ecol. Evol. 28:110-118.

Schnabel, G., Amiri, A., and Brannen, P. M. 2012. Field kit and internet-supported fungicide resistance monitoring. Pages 116-132 in: Fungicide Resistance in Crop Protection: Risk and Management. T. S. Thind, ed. CABI, Oxford.

Sierotzki, H., Blum, M., Olaya, G., Waldner, M., Cohen, Y., and Gisi, U. 2011 Sensitivity to CAA fungicides and frequency of mutations in cellulose synthase 3 (CesA3) gene of oomycete pathogen populations. Pages 151-154 in: Modern Fungicides and Antifungal Compounds. H. Dehne, H. Deising, U. Gisi, K. Kuck, P. Russell, and H. Lyr, eds. Selbstverlag, Braunschweig, Germany.

Sun, H., Wang, H., Stammler, G., Ma, J., Liu, J., and Zhou, M. 2010. Sensitivity of Chinese isolates of Plasmopara viticola to metalaxyl and dimethomorph. J. Phytopathol. 158:450-452.

Tian, S., Su, H., and Nie, S. 2019. Analysis on the production and market situation of Chinese table grape in 2018. Sino-Overseas Grapevine and Wine 2:95-98.

Van Hoeyveld, E., Houtmeyers, F., Massonet, C., Moens, L., Van Ranst, M., Blanckaert, N., and Bossuyt, X. 2004. Detection of single nucleotide polymorphisms in the mannose-binding lectin gene using minor groove binder-DNA probes.J. Immunol. Methods 287:227-230.

Waard, M. A., Georgopoulos, S. G., Hollomon, D. W., Ishii, H., Leroux, P., Ragsdale, N. N., and Schwinn, F. J. 1993. Chemical control of plant diseases: problems and prospects. Annu. Rev. Phytopathol. 31:403-421.

Wong, F. P., and Wilcox, W. F. 2000. Distribution of baseline sensitivities to azoxystrobin among isolates of Plasmopara viticola. Plant Dis. 84:275-281.

Xin, Z., Velten, J. P., Oliver, M. J., and Burke, J. J. 2003. High-throughput DNA extraction method suitable for PCR. Biotechniques 34:820-826.

Yin, L., Zhang, Y., Hao, Y., and Lu, J. 2014. Genetic diversity and population structure of Plasmopara viticola in China. Eur. J. Plant Pathol. 140: 365-376.

Yu, Y., Zhang, Y., Yin, L., and Lu, J. 2012. The mode of host resistance to Plasmopara viticola infection of grapevines. Phytopathology 102:1094-1101.

Zhang, H., Kong, F., Wang, X., Liang, L., Schoen, C. D., Feng, J., and Wang, Z. 2017. Tetra-primer ARMS PCR for rapid detection and characterisation of Plasmopara viticola phenotypes resistant to carboxylic acid amide fungicides. Pest Manag. Sci. 73:1655-1660. 